\title{
VOLUMETRIC EFFECTS DUE TO ION-SOLVENT INTERACTION IN AQUEOUS ELECTROLYTE SOLUTIONS
}

\author{
by \\ TORBEN GRAVES PEDERSEN" and AASE HVIDT ${ }^{21}$ \\ "Department of Chemistry, Carlsberg Laboratory, \\ Gamle Carlsberg Vej 10, DK-2500 Copenhagen Valby \\ ${ }^{23}$ Department of Chemistry, H.C. Ørsted Institute, \\ Universitetsparken 5, DK-2100 Copenhagen Ø
}

Keywords: Chlorides, water, volume, solvation, cation-water, electrostriction

\begin{abstract}
The apparent volume of a series of chlorides dissolved in water - including hydrochloric acid and chlorides of divalent cations - is calculated from available data on the density of the aqueous solutions.

The concentration dependence of the volume of the electrolytes illustrates the long range electrostriction of water by the ions. The volume of the cations, at infinite dilution, reveals that the electrostriction observed, is composed of two opposite volume effects. The experimental data supports the view that bulky, "icelike" water structures predominate near the surface of a small ion, and dense structures, less organized than bulk water, are present at a distance from the ionic center.
\end{abstract}

\section{INTRODUCTION}

Volumetric properties of aqueous solutions of alkali halides were considered in a previous paper (11). Available experimental data indicates that a progessive hydration of the ions in aqueous solution occurs upon dilution, giving rise to negative volume changes (electrostriction of water). A peculiar behaviour of the $\mathrm{Li}^{+}$-salts, compared with other alkali halides, was tentatively associated with the small size of the $\mathrm{Li}^{+}$-ion compared with a water molecule.

The aim of the present paper is to further elucidate the volumetric behavior of aqueous electrolyte solutions. The volume of a series of chlorides, including $\mathrm{HCl}$ and chlorides of divalent cations, is discussed in relation to the concentration of the electrolytes and the radius of the cation.

In ref. 11 the hypothetical state of the molten salts at room temperature was chosen as a reference. The volume in the molten state was estimated by extrapolation from data on the density at higher temperatures. A similar procedure for $\mathrm{HCl}$ is not described in the literature, and in the present paper, the volume of the electrolytes in solution has therefore been compared with the so-called intrinsic volume of the ions calculated from the ionic radius, $R$, as $4 \pi R^{3} / 3$. This intrinsic volume is different from the hypothetical volume of molten salts at room temperature used in ref. 11 and for this reason the extrinsic volume used in the present paper differs from the excess volume used previously.

\section{THE VOLUME OF A SERIES OF CHLORIDES}

The apparent molar volume of solute, $V_{1}^{\text {app }}$, is calculated from the density of a solution in the following way

$$
V_{1}^{a p p}=M_{1}\left[1 / d_{0}^{*}+\left(1 / d-1 / d_{o}^{*}\right) / w_{1}\right]
$$

In this expression $\mathrm{d}$ is the density. The subscripts 


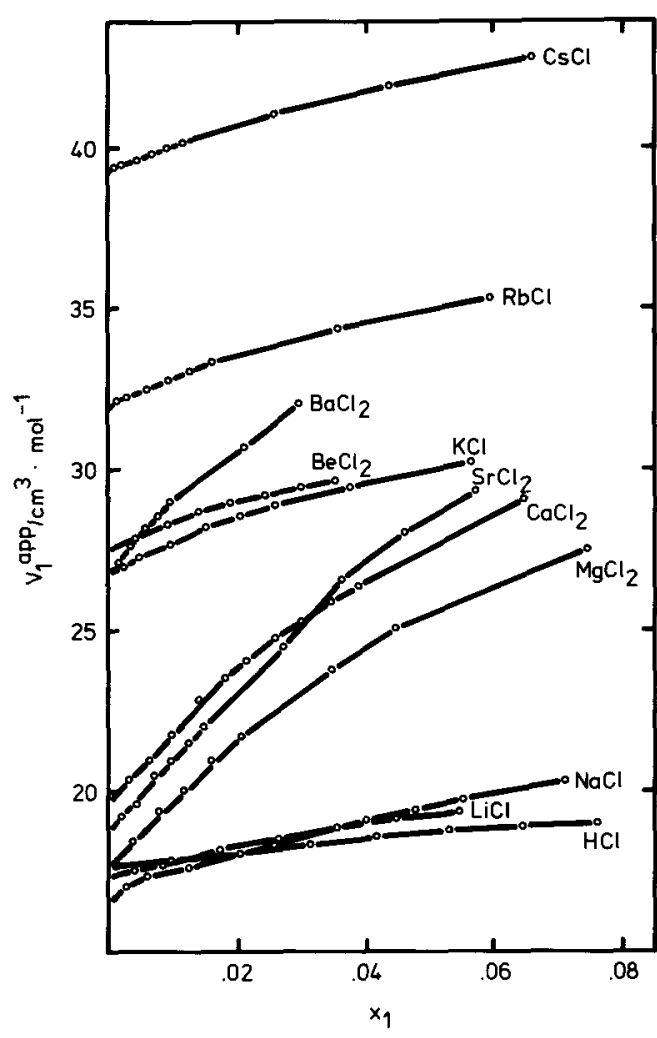

Figure 1. The apparent molar volume of chlorides in aqueous solution at $291 \mathrm{~K}$, calculated from available data (5) according to eqn. (1), $x_{1}$ is the mole fraction of the electrolyte.

\section{Table I}

The radius, $R(\AA)$, taken from ref. 1 , of the cations, and the intrinsic molar volume, $V_{1}^{\text {intr }}\left(\mathrm{cm}^{3} \mathrm{~mole}^{-1}\right)$, of their chlorides.

\begin{tabular}{lrll}
\hline \multicolumn{2}{l}{$\mathrm{R}$ of cation } & \multicolumn{2}{l}{$\mathrm{V}_{1}^{\text {intr }}$ of chlorides } \\
\hline $\mathrm{H}^{+}$ & 0 & $\mathrm{HCl}$ & 14.98 \\
$\mathrm{Li}^{+}$ & .68 & $\mathrm{LiCl}$ & 15.77 \\
$\mathrm{Na}^{+}$ & .98 & $\mathrm{NaCl}$ & 17.36 \\
$\mathrm{~K}^{+}$ & 1.33 & $\mathrm{KCl}$ & 20.94 \\
$\mathrm{Rb}^{+}$ & 1.48 & $\mathrm{RbCl}$ & 23.14 \\
$\mathrm{Cs}^{+}$ & 1.69 & $\mathrm{CsCl}$ & 26.76 \\
$\mathrm{Be}^{2+}$ & .30 & $\mathrm{BeCl}_{2}$ & 30.02 \\
$\mathrm{Mg}^{2+}$ & .65 & $\mathrm{MgCl}_{2}$ & 30.65 \\
$\mathrm{Ca}^{2+}$ & .94 & $\mathrm{CaCl}_{2}$ & 32.05 \\
$\mathrm{Sr}^{2+}$ & 1.10 & $\mathrm{SrCl}_{2}$ & 33.32 \\
$\mathrm{Ba}^{2+}$ & 1.29 & $\mathrm{BaCl}_{2}$ & 35.38 \\
\hline
\end{tabular}

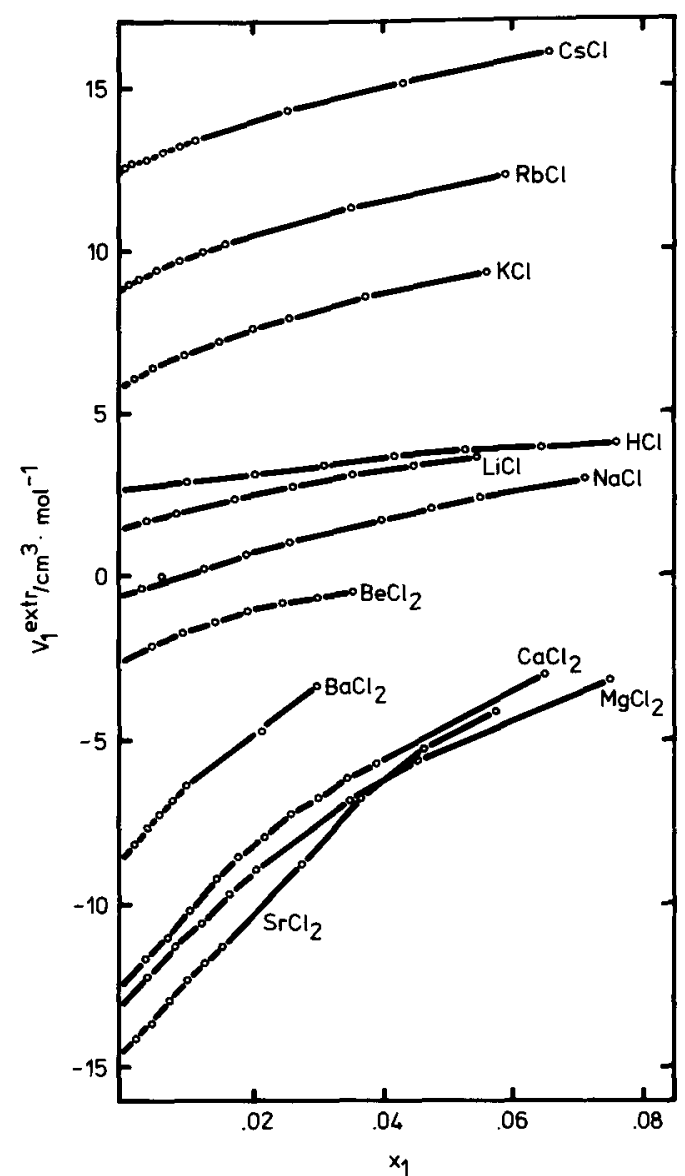

Figure 2. The extrinsic molar volume of chlorides in aqueous solution at $291 \mathrm{~K}, \mathrm{~V}_{1}^{\text {extr }}=\mathrm{V}_{1}^{\mathrm{app}}-\mathrm{V}_{1}^{\mathrm{intr}}$. $\mathrm{V}_{1}^{\mathrm{app}}$ is from Figure 1, and $V_{1}^{\mathrm{intr}}=4 \pi R^{3} / 3$, where $R$ is the ionic radius, reported in Table l. $x_{1}$ is the mole fraction of the electrolyte.

0 and 1 refer to the solvent and the solute, respectively; $w$ is weight fraction, and the superscript* denotes the pure liquid state.

Figure 1 illustrates the concentration dependence of $\mathrm{V}_{1}^{\text {app }}$ of a series of chlorides, calculated according to eqn. (1) from data interpolated to $291 \mathrm{~K}$ from avaible density measurement on aqueous solutions (5).

Table $I$ reports the radius of the cations considered and the intrinsic molar volume of their chlorides.

Figure 2 shows the concentration dependence of the extrinsic volume of the electrolytes, de- 


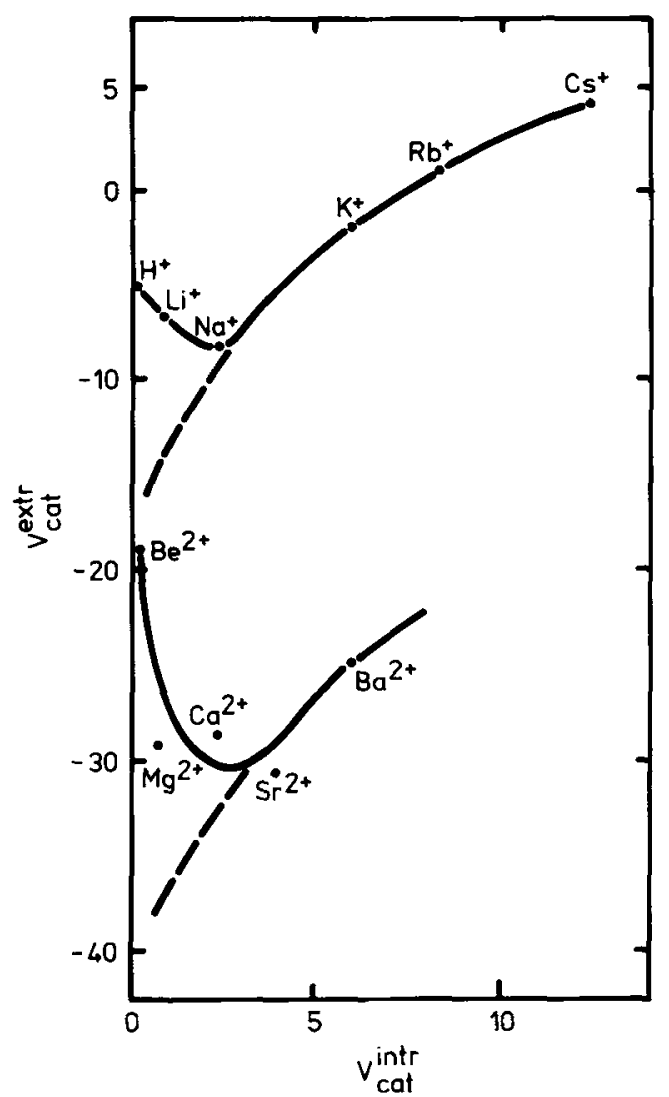

Figure 3. The extrinsic molar volume of cations at infinite dilution in aqueous solution at $291 \mathrm{~K}$. The abscissa is the intrinsic volume of the cations. $\left(\mathrm{cm}^{3} \cdot \mathrm{mol}^{-1}\right)$.

fined as the difference between the apparent volume in solution and the intrinsic volume, $\mathrm{V}_{1}^{\text {extr }}=\mathrm{V}_{1}^{\text {app }}-\mathrm{V}_{1}^{\mathrm{intr}}$.

The molar volume of the chloride ion, at infinite dilution, is $23,0 \mathrm{~cm}^{3} \mathrm{~mol}^{-1}(6,10)$, and the intrinsic volume is $15,0 \mathrm{~cm}^{3} \mathrm{~mol}^{-1}$ (see Table I). The extrinsic volume of the chloride ion, $\mathrm{V}_{\mathrm{Cl}^{-}}^{\text {ext }}=$ $23.0-15.0=8.0 \mathrm{~cm}^{3} \mathrm{~mol}^{-1}$, is subtracted from the extrinsic volume of the salts, at infinite dilution (Figure 2), in order to give the extrinsic volume of the cations. This volume is presented in Figure 3 as a function of the intrinsic volume.

\section{DISCUSSION}

Electrostriction of a liquid is defined as the volume change caused by an electric field $(1,8)$.

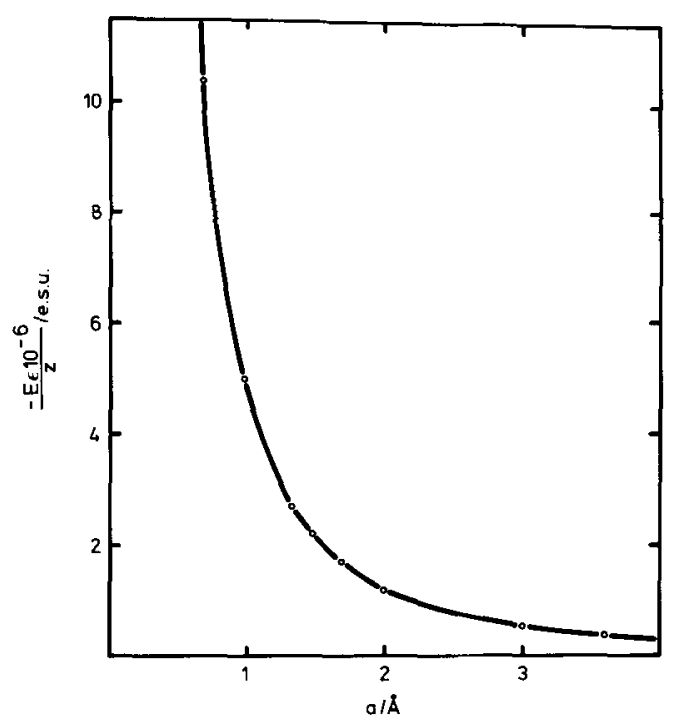

Figure 4. The strength of the electrostatic field around an ion, calculated according to eqn. (2). $a$ is the distance from the center of the ion.

The field strength around an ion may be expressed as

$$
\mathrm{E}=-\mathrm{q} / \varepsilon \mathrm{a}^{2},
$$

where $\mathrm{q}$ is the ionic charge $\left(\mathrm{q}=\mathrm{Z} 4.810^{-10}\right.$ e.s.u. $)$, $\varepsilon$ is the dielectric constant of the medium, and a is the distance from the center of the ion. Figure 4 illustrates the variation of the field strength with the distance from the charge. The increase of the strength with decreasing values of $a$ is remarkable in the range relevant of the interaction of an ion with neighbouring water molecules, and for this reason an increase of the electrostriction of water, with decreasing ion radius, might be anticipated.

The interaction of the electrostatic field of an ion with water tends to align the dipolar water molecules in the direction of the field. In this way the field tends to disrupt hydrogen bonded structures in liquid water, and to compress the water molecules surrounding an ion. These electrostatic effect give rise to a shrinkage of the water. The extent of the alignment of the water molecules depends on the ratio $\mu \mathrm{E} / \mathrm{kT}$, where $\mu$ is the dipole moment of water, $\mu=1.83$ Debye. The alignment is perfect if $\mu \mathrm{E}>>\mathrm{kT}$, i.e. if $\mathrm{E}>>210^{4}$ e.s.u. $\left(610^{6} \mathrm{Vcm}^{-1}\right)$. This condition is only fulfilled for the proton, but a consideration 


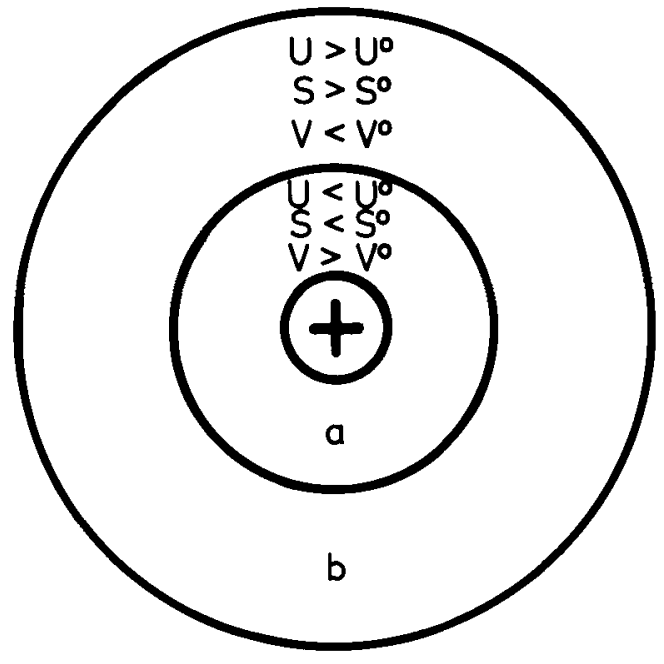

bulk water $\left(U^{0}, S^{0}, V^{0}\right)$

Figure 5. Model for structure modifications of water produced by a small cation. $U, S$ and $V$ stands for molar energy, entropy and volume, respectively.

of eqn. (2), or of Figure 4, shows that very high electric fields do exist near the surface of the smaller ions.

There can hardly be any doubt that water molecules are most strongly attracted to, and oriented towards the smaller ions. However, Figure 3 demonstrates the very fact that a positive contribution to the electrostriction is observed for the smaller ions. This positive volume effect is illustrated in Figure 3 by the difference between the fully drawn curve and the dashed extrapolation. The experimental data thus brings up the question whether the strong attraction between a small ion and water is compatible with a positive volume change.

In the case of the $\mathrm{Li}^{+}$-ion the positive volume effect (about $7 \mathrm{~cm}^{3} \mathrm{~mole}^{-1}$, see Figure 3) was in ref. 11 tentatively ascribed to a void volume between the ion and the first shell of surrounding water molecules. The corresponding effect observed for $\mathrm{H}^{+}$and for $\mathrm{Be}^{++}$is, however, so large, that this explanation is insufficient to explain the observations.

According to the model of structure modifications of water by small ions, originally proposed by FRANK and coworkers $(3,4)$, an ion dissolved

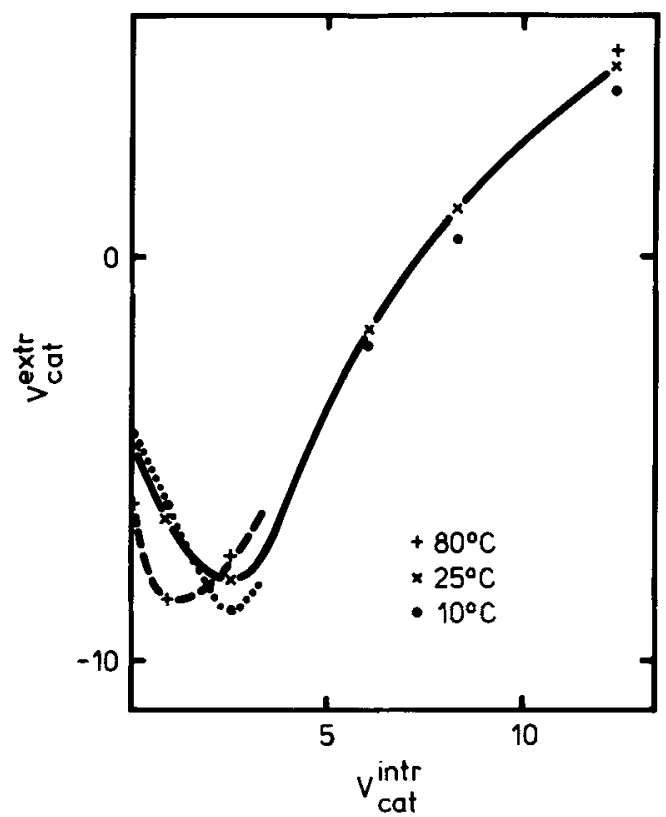

Figure 6. The extrinsic molar volume of monovalent cations in aqueous solutions at infinite dilution at 283 , 298 and $353 \mathrm{~K}$, calculated from available data (5). The abscisse is the intrinsic molar volume of the cations. $\left(\mathrm{cm}^{3} \cdot \mathrm{mol}^{-1}\right)$.

in water is surrounded by two concentric regions of structurally different types of water. Water molecules in the innermost region (a) are assumed to be immobilized, whereas molecules in the outermost region (b) are even more randomly organized than the molecules in bulk water.

On the basis of this suggested picture of structured water the present volumetric data indicates (see Figure 5) that region $a$ of water (characterized by low energy and entropy) has a larger, and region $b$ (characterized by high energy and entropy) has a smaller specific volume than pure water.

This volumetric behaviour is in accordance with the unusual volumetric properties of water, such as the unique temperature dependence of the volume of liquid water (the negative expansivity below $4^{\circ} \mathrm{C}$ ), or the expansion accompanying the freezing of liquid water to ice, and the structural changes of water introduced by nonpolar solutes (7). The temperature dependence of the extrinsic volume of monovalent cations 
(5), illustrated in Figure 6, supports the view that bulky, "icelike" water structures surrounding the smaller ions "melt" upon heating.

These peculiar volume effects are associated with hydrogen bonding between water molecules. They reflect the fact that although hydrogen bonding diminishes the distance between neighbouring water molecules, it gives rise to an expansion, due to the directional character of the hydrogen bond. Hydrogen bonding decreases the number of nearest neighbours to a given water molecule, and in this way relatively large void spaces are created between the molecules (2). In ice the coordination number of the water molecules is 4; the apparent volume of small cations in aqueous solution suggests that tetrahedrical ("icelike") water structures exist in the immediate surroundings of the ions. Convincing experimental evidence is available that a proton binds a water molecule in a proton-water complex, $\mathrm{H}_{3} \mathrm{O}^{+}$, which is coordinated through hydrogen bonding with three or four water molecules in an open structure (1).

The over-all negative electrostriction of water originates from the dielectric polarization of more distant water molecules (region b). Figures 1 and 2 illustrate how the extent of this effect increases upon dilution. The absence of any inflection point of the volume curves, even at the lowest electrolyte concentrations studied $(x \simeq$ $10^{-3}$ ), reflects the electrostriction as an extremely long range effect.

In summary, the suggested model of water structures around an ion as composed of two distinguishable regions is undoubtedly a crude oversimplification of the conditions in an electrolyte solution, but it elucidates essential features of the solute-solvent interaction. The structural changes of water, introduced by an ion, occur as a result of two competing orienting influences on any water molecule. One is the influence from neighbouring water molecules; the other is the influence from the spherically symmetrical ionic field. The first is present in bulk water, and the latter predominates near the surface of a small, or multiply charged ion. In between there is a region of more orientational disorder than exists in pure water. The volumetric data, here discussed, supports this picture of water, taking into consideration the character- istic thermodynamics of structural equilibria in liquid water. The formation of ordered, lowenergy structures among water molecules is accompanied by an expansion $(\Delta \mathrm{H} \cdot \Delta \mathrm{V}<0$, and $\Delta \mathrm{S} \cdot \Delta \mathrm{V}<0$ ). The long range structural changes of water introduced by ions, and drastically reflected in volumetric properties, may play an essential role in cell physiology, particularly in the mechanism of the $\mathrm{Na}^{+}$pump, extensively discussed by LING (9).

\section{ACKNOWLEDGEMENTS}

We wish to thank professor MARTIN OTTESEN for his critical review of the manuscript, and Christian Dethlefsen for valuable discussions. LIZZIE AUGUSTSEN is gratefully acknowledged for drawing the figures.

\section{REFERENCES}

1. Conway. B. E.: Ionic hydration in chemistry and biophysics. Elsevier, Amsterdam (1981)

2. EdSAll. J. T. \& H. A. MCKenZIE: Water and proteins. II The location and dynamics of water in protein systems and its relation to their stability and properties. Adv. Biophys., Vol 16, 53-183 (1983)

3. Frank, H. S. \& M. W. Evans: Free volume and entropy in condensed systems. III Entropy in binary liquid mixtures; Partial molal entropy in dilute solutions; Structure and thermodynamics in aqueous electrolytes. J. Chem. Phys. 13, 507-532 (1945)

4. FRANK, H. S. \& W. WEN: III. Ion-solvent interaction. Structural aspects of ion-solvent interaction in aqueous solutions: A suggested picture of water structure. Disc. Far. Soc. 24, 133-140 (1957)

5. GILLESPIE, L. J.: Density (specific gravity) and thermal expansion (under atmospheric pressure) of aqueous solutions of inorganic substances and of strong electrolytes. In: E. W. Washburn, ed., International Critical Tables of Numerical Data, Physics, Chemistry and Technology, McGrawHill, New York, Vol. 3, 54-95 (1928)

6. HORNE, R.A.: Water and aqueous solutions. Structure, thermodynamics and transport processes. Wiley-Interscience, Toronto (1972)

7. HVIDT, AA.: Interaction of water with nonpolar solutes. Ann. Rev. Biophys. Bioeng. 12, 1-20 (1983)

8. Linderstrøm-LANG, K. \& C. F. Jacobsen: The concentration accompanying enzymatic break- 
down of proteins. Compt. rend. trav. lab. Carlsberg Ser. Chim. 24, 1-48 (1941)

9. LING, G. N.: In search of the physical basis of life. Plenum Press, New York and London (1984)

10. Millero, F. J.: The molal volumes of electrolytes.
Chemical Reviews 71(2), 147-176 (1971)

11. Pedersen, T. G., C. Dethlefsen \& Aa. Hvidt: Volumetric properties of aqueous solutions of alkali halides. Carlsberg Res. Commun. 49, 445455 (1984)

Accepted by H. KLENOW 